\title{
Estimating Academic Attrition from Technical Training School Data: Method and Simulation Results
}

Tirso Diaz

Michael Ingerick

Robert Fowler

Mary Ann Lightfoot

Human Resources Research Organization

United States Army Research Institute for the Behavioral and Social Sciences

\section{August 2004}

Approved for public release: distribution is unlimited 